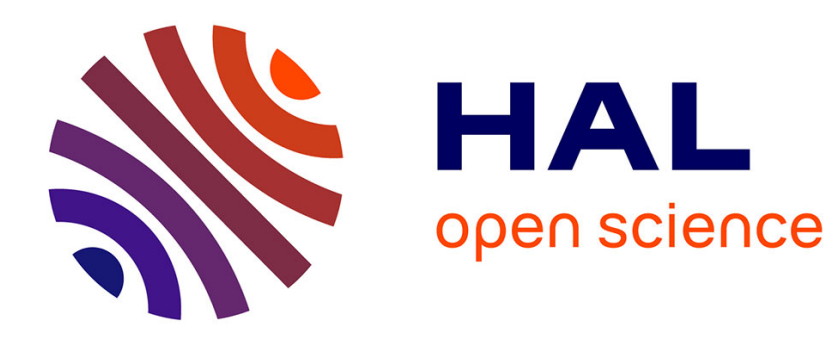

\title{
Three-Magnon Decay of Exchange Spin Wave
}

A. Temiryazev, M. Tikhomirova

\section{To cite this version:}

A. Temiryazev, M. Tikhomirova. Three-Magnon Decay of Exchange Spin Wave. Journal de Physique IV Proceedings, 1997, 07 (C1), pp.C1-391-C1-392. 10.1051/jp4:19971158 . jpa-00254803

\section{HAL Id: jpa-00254803 https://hal.science/jpa-00254803}

Submitted on 1 Jan 1997

HAL is a multi-disciplinary open access archive for the deposit and dissemination of scientific research documents, whether they are published or not. The documents may come from teaching and research institutions in France or abroad, or from public or private research centers.
L'archive ouverte pluridisciplinaire HAL, est destinée au dépôt et à la diffusion de documents scientifiques de niveau recherche, publiés ou non, émanant des établissements d'enseignement et de recherche français ou étrangers, des laboratoires publics ou privés. 


\title{
Three-Magnon Decay of Exchange Spin Wave
}

\author{
A.G. Temiryazev and M.P. Tikhomirova \\ Institute of Radioengineering \& Electronics, Vvedenskii sq. 1, Fryazino, Moscow Region 141120, Russia
}

\begin{abstract}
Noninear properties of short-wavelength exchange-dominated spin waves in ferrite films have been studied experimentally. Collinear splitting of exchange spin waves into two waves has been observed. An yttrium-iron-garnet film with smooth variation of the uniaxial anisotropy field through the film thickness has been used for the experimental study. Nonuniformity of the film provided both the linear excitation of short-wavelength pumping wave and the detection of the radiation caused by the parametrically excited waves.
\end{abstract}

It is well known that the first-order Suhl instability can be explained in terms of three magnon decay. Linear excitation of a pumping wave at frequency $\omega_{p}$ causes the parametrical excitation of a pair of spin waves at frequencies $\omega_{1}$ and $\omega_{2}$ which satisfy $\omega_{p}=\omega_{1}+\omega_{2}$. Up to now, the low-wavenumber spin waves (so-called magnetostatic waves with wavenumbers $q_{p} \leq 10^{3}$ $\mathrm{cm}^{-1}$ ) have been used as a pumping wave. Representative $q$-values of the parametrically excited waves are about $10^{4}-10^{5}$ $\mathrm{cm}^{-1}$. Thus, $q_{p} \ll q_{1,2}$, and the conservation law of momentum $\left(q_{p}=q_{1}+q_{2}\right)$ is fulfilled if $q_{1} \approx-q_{2}$. The three magnon decay of the low-wavenumber spin waves is possible at frequencies $\omega_{p} \geq 2 \gamma H_{i n t}$, where $H_{i n t}$ is the static internal magnetic field.

We have considered the case when the pumping wave is a higlh-wavenumber wave and the condition $q_{p}>q_{1,2}$ is fulfilled. A short-wavelength exchange-dominated spin wave (ESW) was used as a pumping wave. It can be obtained from the dispersion law of ESW and the conservation laws that the decay is possible if $\omega_{p} \geq 3 \gamma H_{\text {int }}$. The limiting frequency $\omega_{p}=$ $3 \mu H_{\text {int }}$ corresponds to the case of frequency-degenerate collinear decay, with $\omega_{1}=\omega_{2}=\omega_{p} / 2$, and $\mathbf{q}_{1}=\mathbf{q}_{2}=\mathbf{q}_{p} / 2$. It can be shown that only sufficiently short waves, with $q_{p}{ }^{2} \geq 2 \omega_{p} / 3 \gamma D$, where $D$ is the exchange stiffness, can decay. Even at a rather low pumping frequency $\omega_{p} / 2 \pi=1 \mathrm{GHz}$, the wave number $q_{p}$ must exceed $2.2 \cdot 10^{5} \mathrm{~cm}^{-1}$. Hence, the parametrically excited waves must also have large values of $q\left(q_{1} \approx q_{p} / 2\right)$.

It follows that an experimental study would require the capability to excite and receive exchange spin waves with $q \sim$ $10^{5} \mathrm{~cm}^{-1}$, in order to (first) create the pump wave and (second) detect the decay products. This problem can be solved by working with nonuniform yttrium iron garnet (YIG) films [1,2]. To explain the experiment, let us consider a YIG film in an external magnetic field $\boldsymbol{H}$ which is directed at an angle $\theta$ to the $\mathrm{x}$-axis perpendicular to the plane of the film. Assume that
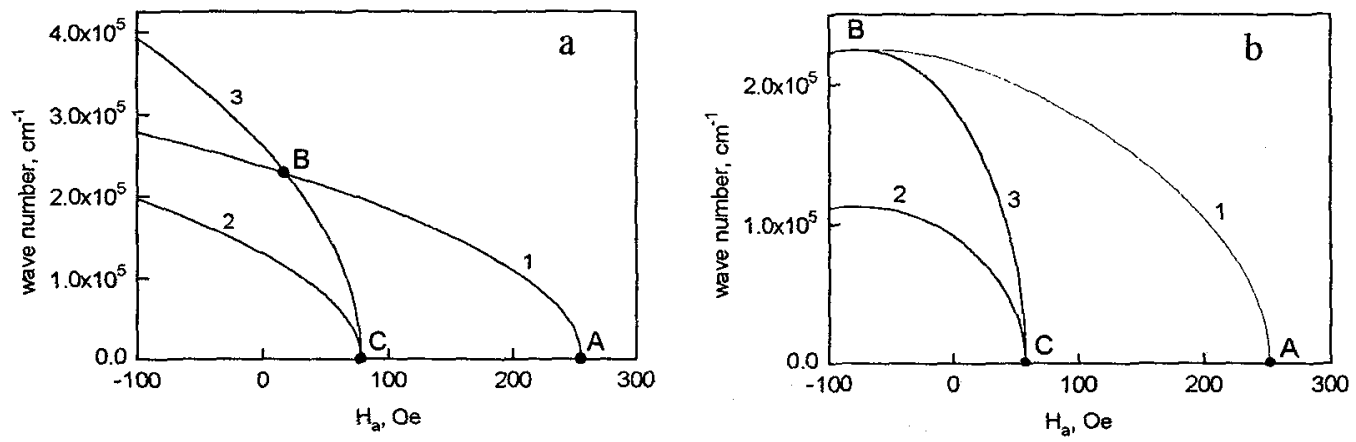

Figure 1: Wave number versus the anisotropy field for a film with $4 m M_{\text {sat }}=1750 . \mathrm{G}$ in the bias magnetic field of $H=1850 \mathrm{Oe}$. (1) $-q\left(H_{a}\right)$ at $\omega / 2 \pi=1000 \mathrm{MHz} ;(2)-q\left(H_{a}\right)$ at $\omega / 2 \pi=500 \mathrm{MHz}$; (3) $-2 q\left(H_{a}\right)$ at $\omega / 2 \pi=500 \mathrm{MHz}$ (a) $-\theta=0,(\mathrm{~b})-\theta=0.56^{\circ}$ 

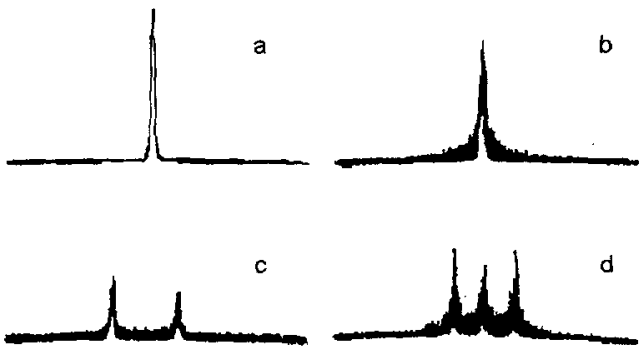

Figure 2: Typical emission spectra. The power applied to the sample was $80 \mathrm{~mW}$. The various spectra correspond to various strengths and directions of the extemal field. For all spectra, the horizontal sweep is $100 \mathrm{kHz}$, and the central frequency is $500 \mathrm{MHz}$. The gain of the receiver for spectrum (a) is $20 \mathrm{~dB}$ lower than that for spectra (b)-(d).

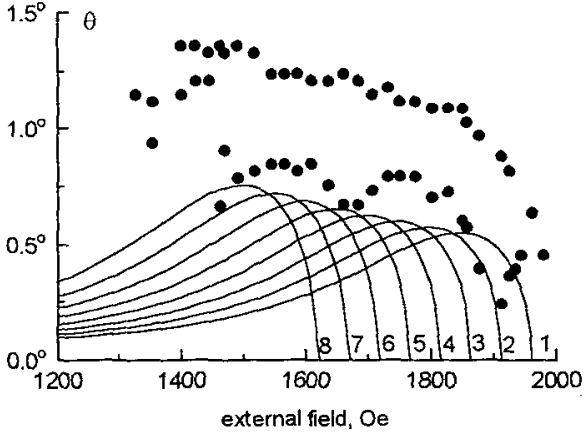

Figure 3: Points - experimental values of $\theta_{\max }$ and $\theta_{\min }$; curves - theoretical values of $\theta_{i}(H)$ for layers with $H_{a}=-100+50(i-1) \mathrm{Oe}$. The curve label is the value of $i$.

anisotropy field $H_{a}$ depends on $x$, while the other parameters of the film are constants. For $\theta=0$, the profile of the wave number along the coordinate $x$ or the dependence of this wave number on the anisotropy field $H_{a}$ can be found from the dispersion relation $\omega / \gamma=H-4 \pi M_{\text {sat }}+H_{a}(x)+D q^{2}(x)$, where $M_{\text {sat }}$ is the saturation magnetization. For $\theta \neq 0$, such calculations can be conducted numerically. Figure 1 shows plots of $q_{p}\left(H_{a}\right)$ at $\omega_{p} / 2 \pi=1000 \mathrm{MHz}$ (curve 1 ), of $q_{1}\left(H_{a}\right)$ at $\omega_{1}=\omega_{p} / 2$ (curve 2 ), and $2 q_{1}\left(H_{a}\right)$ (curve 3 ). At point $A$, where $q_{p} \approx 0$, the pump wave is excited [1,2]. The conditions for degenerate collinear decay are satisfied at point $B$. When the products of this decay reach point $C$, they have a $q_{1}$ on the order of zero, so we can expect radiation of electromagnetic waves with a frequency $\omega_{1}$ from the sample. To the left of point $B$, where we have $2 q_{1}>$ $q_{p}$, there can be a parametric excitation of frequency-nondegenerate waves. The occurrence of this process can be inferred from the occurrence of emission at frequencies differing from $\omega_{p} / 2$.

For the experiments we used a (100) YIG film with a thickness of $11 \mu \mathrm{m}$. The field $H_{a}(x)$ varied smoothly from 250 Oe at one surface to $-100 \mathrm{Oe}$ in a uniform layer $2 \mu \mathrm{m}$ thick adjacent to the second surface. The film is pressed against a stripline $50-500 \mu \mathrm{m}$ wide, to which a microwave signal with a frequency $\omega_{p} / 2 \pi=1000 \mathrm{MHz}$ is applied. Emission from the sample is detected by the same stripline and then sent through a directional coupler to a spectrum analyzer. The spectrum of this emission was studied for various strengths and directions of the external magnetic field.

It was found that, as the power applied to the sample is raised to $\sim 1 \mathrm{~mW}$, signals with frequencies close to $\omega_{p} / 2$ appear in the spectrum. Fig. 2 shows the typical spectra of radiation. Let us highlight some of the experimental results.

1. The spectra in Fig. 2, (a) and (b), demonstrate the possibility of a parametric excitation of spin waves with a frequency equal to precisely half the pump frequency, while the spectra in (c) and (d), show that the decay can be frequencynondegenerate (c), and that it can also occur simultaneously in several channels (d).

2. The emission spectrum can be extremely narrow (Fig. 2a). Then, the observed linewidth is determined by the resolution $(1 \mathrm{kHz})$ of the spectrum analyzer, and there are no significant traces of noise in the emission. In this case the emission intensity is high; it can reach $100 \mu \mathrm{W}$ at an input power of $80 \mathrm{~mW}$.

3. Emission was observed only when $\theta \neq 0$. The interval $\theta_{\min } \leq \theta \leq \theta_{\max }$ in which the emission was detected was extremely narrow, about $1^{\circ}$. Fig. 3 shows values of $\theta_{\min }$ and $\theta_{\max }$ for various values of $H$.

Some results can be explained. The experimental setup allows to detect only the products of a collinear decay, when $q_{p}, q_{1}$, and $q_{2}$ are directed along the $x$-axis. The threshold for the collinear decay goes off to infinity if all three wave vectors are oriented parallel to the magnetization [3]. It explains why we see no radiation at $\theta=0$. We have calculated the dependence $\theta_{i}(T)$ for which conservation laws hold for the degenerate collinear decay of ESW which is propagating along the $x$-axis. Fig. 3 shows that at large values of $\theta$ the conservation laws are not fulfilled at any value of $H_{a}$. Thus, the decay is possible in a very narrow interval of angles.

\section{Acknowledgments}

We gratefully acknowledge financial support by the Russian Foundation for Basic Research (Grant No. 94-02-04928-a). We are thankful to A. V. Maryakhin for providing us with the YIG films.

\section{References}

[1]. Teniryazev A.G., Tikhomirova M.P., and Zil'berman P.E., J. Appl. Phys 76 (1994) 5586-5588.

[2]. Zil'berman P.E., Temiryazev A.G., and Tikhomirova M.P., Sov. JETP 81 (1995) 151-162.

[3]. L'vov V.S., Nonlinear Spin Waves (Nauka, Moscow, 1987) p. 32. 